\title{
Evaluación de propiedades antioxidantes y fisicoquímicas de una bebida mixta durante almacenamiento refrigerado
}

\author{
Antioxidant and physicochemical properties evaluation of a mixed beverage during \\ refrigerated storage
}

Margareth Santander-M. ${ }^{1}$; Oswaldo Osorio M. ${ }^{2}$; Diego Mejía-España ${ }^{3}$.

1 Ingeniera Agroindustrial. Centro de Investigación Tibaitatá, Corporación Colombiana de Investigación AgropecuariaCORPOICA, Bogotá, Colombia, marguissantanderm@gmail.com.

2 Profesor Titular. Ingeniero Agroindustrial. Ph. D. Grupo de Apoyo a la Investigación y Desarrollo Agroalimentario (GAIDA). Universidad de Nariño, Pasto, Colombia, osorio_oswaldo@hotmail.com.

3 Profesor Asistente. Ingeniero Agroindustrial. M.Sc. Grupo de Apoyo a la Investigación y Desarrollo Agroalimentario (GAIDA). Universidad de Nariño, Pasto, Colombia, diegomejiaespana@gmail.com.

Citar: SANTANDER-M, M.; OSORIO, 0.; MEJIA-ESPAÑA, D. 2017. Evaluación de propiedades antioxidantes y fisicoquímicas de una bebida mixta durante almacenamiento refrigerado. Rev. Cienc. Agr. 34(1): 84-97. doi: http:// dx.doi.org/10.22267/rcia.173401.65.

Recibido: Marzo 03 de $2016 . \quad$ Aceptado: Octubre 16 de 2016.

\section{RESUMEN}

En la actualidad, los consumidores tienden a adquirir alimentos funcionales, los cuales satisfacen los requerimientos nutricionales y adicionalmente aportan beneficios a la salud. El creciente interés en el estudio de bebidas con estas características ha contribuido al desarrollo de productos elaborados a partir de la mezcla de leche y frutas. El objetivo de esta investigación fue evaluar una bebida mixta de leche UHT descremada con pulpa de tomate de árbol, analizando sus características antioxidantes y fisicoquímicas, mediante la determinación del contenido de ácido ascórbico, compuestos fenólicos, carotenoides y su estabilidad durante el almacenamiento bajo refrigeración. La capacidad antioxidante se estudió a través del método DPPH, el ácido ascórbico y los carotenoides fueron analizados mediante cromatografía líquida de alto rendimiento y se empleó el método de Folin-Ciocalteu para determinar fenoles totales en la bebida. Se encontró que la bebida mixta presentó compuestos bioactivos reconocidos por sus propiedades funcionales. Durante las primeras dos semanas de almacenamiento refrigerado se observó una disminución significativa $(\mathrm{P}<0,05)$ del contenido de compuestos bioactivos. Hubo una reducción en el contenido de fenoles totales de 33,150 $\pm 2,020$ a 21,467 11,360mg equivalentes de ácido gálico (GAE)/100mL, al igual que en la concentración de ácido ascórbico de $12,967 \pm 0,603$ a $5,167 \pm 0,321 \mathrm{mg} / 100 \mathrm{~mL}$ y en el de $\beta$-caroteno de $243,00 \pm 5,66$ a $146,50 \pm 2,12 \mu \mathrm{g} / 100 \mathrm{~mL}$, 
lo cual se reflejó en la disminución de la capacidad antioxidante de la bebida en un rango de 3,450 $\pm 0,041$ a 1,581 $\pm 0,025$ de Trolox mmol/L. El pH y los sólidos solubles totales se mantuvieron constantes, mientras que la acidez aumentó significativamente durante la primera semana de almacenamiento.

Palabras clave: Tomate de árbol, frutas, leche, ácido ascórbico, compuestos fenólicos, carotenoides.

\begin{abstract}
Consumers tend to buy functional foods which meet the nutritional requirements and additionally provide health benefits. The growing interest in the study of beverages with these characteristics has contributed to the development of products based on mixture of milk and fruits. The objective of this research was to evaluate a mixed beverage based on UHT milk and pulp of tamarillo fruit, analyzing its antioxidant and physicochemical characteristics, by determining the content of ascorbic acid, phenolic compounds, carotenoids and their stability during refrigeration. The antioxidant capacity was studied through the DPPH method, ascorbic acid and carotenoids were determined by high performance liquid chromatography and the Folin-Ciocalteu method was used to analyze total phenols in the beverage. We found that the mixed beverage presented bioactive compounds recognized for their functional properties. A significant decrease in the content of bioactive compounds was observed during the first two weeks of refrigerated storage. There was a decrease in the total phenolic content of $33.150 \pm 2.020$ to $21.467 \pm 1.360 \mathrm{mg}$ gallic acid equivalents (GAE) $/ 100 \mathrm{~mL}$, as in the ascorbic acid content of $12.967 \pm 0.603$ to $5.167 \pm 0.321 \mathrm{mg} / 100 \mathrm{~mL}$ and the $\beta$-carotene content from $243.00 \pm 5.66$ to $146.50 \pm 2.12 \mu \mathrm{g} / 100 \mathrm{~mL}$, which was reflected in the decrease of the antioxidant capacity of the beverage in a range of $3.450 \pm 0.041$ to $1.581 \pm 0.025$ Trolox $\mathrm{mmol} / \mathrm{L}$. During the storage period at $4^{\circ} \mathrm{C}$. The $\mathrm{pH}$ and total soluble solids remained constant, while the acidity increased significantly during the first week of storage.
\end{abstract}

Keywords: Tamarillo fruit, fruit, milk, ascorbic acid, phenolic compounds, carotenoids.

\section{INTRODUCCIÓN}

Hoy en día, hay una clara tendencia hacia el consumo de ciertos alimentos que más allá de su valor nutritivo, contribuyen a mejorar la salud y a reducir el riesgo de enfermedades. Estos productos generalmente se conocen como alimentos funcionales (Rodríguez et al., 2014; Andrés et al., 2014).

De acuerdo con lo anterior, una gran cantidad de alimentos líquidos pasteurizados basados en bebidas de frutas y leche han surgido como productos innovadores que marcan una nueva tendencia en el mercado y han experimentado un rápido crecimiento en los últimos años (Pszczola, 2005; Salvia et al., 2011). Varias investigaciones relacionadas con los alimentos funcionales se han centrado en el estudio de sus propiedades antioxidantes. Un antioxidante es una molécula capaz de retardar 0 prevenir el daño oxidativo de los lípidos, las proteínas y los ácidos nucleicos por especies reactivas del oxígeno, las cuales se generan por causas ambientales o por la ingesta de algún contaminante. Entre esas moléculas que remueven los radicales libres y que se encuentran naturalmente en los alimentos están la vitamina $\mathrm{C}$, los compuestos fenólicos (flavonoides y antocianinas) y carotenoides (el $\beta$-caroteno, fuente de vitamina A) (Castañeda et al., 2008). He et al. (2015) señalan que debido a sus propiedades biológicas pueden prevenir el cáncer, enfermedades cardiovasculares, Alzheimer, diabetes y otros trastornos inducidos por el estrés oxidativo.

El interés en el estudio de bebidas funcionales realizadas con la mezcla de leche y frutas se 
refleja en el desarrollo de investigaciones en torno al análisis de la capacidad antioxidante mediante la determinación del contenido de vitaminas A y C, compuestos fenólicos y carotenos, la bioaccesibilidad in vitro de los anteriores compuestos bioactivos, la aplicación de tecnologías emergentes para su conservación como las altas presiones y campos eléctricos de alta intensidad, los ingredientes, la formulación, la composición fisicoquímica, el valor nutricional y la estabilidad microbiológica (Zulueta et al., 2007; Morales et al., 2010; Salvia et al., 2011; Rodríguez et al., 2014, 2015; He et al., 2015).

Sin embargo, a pesar del potencial que posee el departamento de Nariño para desarrollar este tipo de bebidas no se han realizado investigaciones relacionadas. Tanto la leche como el tomate de árbol son materias primas priorizadas por su importancia socioeconómica, que pueden ser procesadas agroindustrialmente debido a su disponibilidad y a su alto valor nutricional. El tomate de árbol posee compuestos bioactivos entre los que se encuentran la vitamina $C$, compuestos fenólicos y $\beta$-caroteno (Beecher, 1998), mientras que la leche tiene antioxidantes no proteicos como las vitaminas $\mathrm{A}, \mathrm{C}$ y $\mathrm{E}$ y proteicos entre los que se incluyen varios tipos de enzimas y una serie de proteínas y péptidos (Cervato, 1999; Lindmark y Akesson, 2000; Satué et al., 2000).

Por lo anterior, el desarrollo e implementación de productos con características funcionales por parte de empresas agroindustriales es una alternativa para mejorar las condiciones económicas de dos cadenas productivas: la láctea y la hortofrutícola, a través del desarrollo de nuevos productos que generan valor agregado a estas materias primas, impulsando la economía regional y la articulación de los actores involucrados.

El objetivo de esta investigación fue desarrollar una bebida basada en la mezcla de leche UHT descremada y pulpa natural de tomate de árbol estudiando su valor funcional y composición fisicoquímica, a través de la evaluación de sus carac- terísticas antioxidantes mediante el análisis del contenido de ácido ascórbico, compuesto fenólicos, carotenoides y su estabilidad durante el almacenamiento bajo refrigeración.

\section{MATERIALES Y MÉTODOS}

Localización. El presente trabajo se realizó en la Planta Piloto adscrita a la Facultad de Ingeniería Agroindustrial de la Universidad de Nariño, localizada a $1^{\circ} 14^{\prime} 00^{\prime \prime}$ LN y $77^{\circ} 18^{\prime} 00^{\prime \prime}$ LO, a 2506 msnm, con una temperatura promedio de $20^{\circ} \mathrm{C} \mathrm{y}$ humedad relativa de 75\% (Software Android GPS Navigator 2.06).

Muestras. Tomate de árbol (Solanum betaceum (Cav.) Sendt) variedad rojo común y leche UHT descremada $(0,1 \%$ de grasa) fueron adquiridos en un supermercado local. La pulpa de tomate de árbol se extrajo después de aplicar a la fruta un proceso apropiado de lavado e higienización. La bebida se preparó mezclando $30 \%(\mathrm{p} / \mathrm{v})$ de pulpa de tomate con $19 \%(\mathrm{v} / \mathrm{v})$ de leche UHT descremada, $45 \%$ (v/v) de agua, $6 \%$ (p/v) de azúcar y $0,3 \%$ (p/v) de pectina cítrica de alto metoxilo. Los ingredientes sólidos se colocaron en agua en las proporciones de peso indicadas. La bebida se preparó inmediatamente antes de iniciar las determinaciones de las propiedades fisicoquímicas y antioxidantes.

Tratamiento térmico. La bebida fue tratada térmicamente a $90^{\circ} \mathrm{C}$ durante un minuto para asegurar la inactivación de microorganismos de degradación (Salvia et al., 2011). Se emplearon frascos de vidrio estériles twist-off, los cuales se introdujeron en un baño termostatado a temperatura de pasteurización. El choque térmico se realizó empleando un baño de hielo. Una vez obtenidas las muestras, se almacenaron a $4 \pm 1^{\circ} \mathrm{C}$ y en oscuridad hasta los análisis.

Variables fisicoquímicas. El pH se midió con un pH-metro (Tecpel CO Ltd., Taiwán, China), la viscosidad se determinó con un viscosímetro rotacional (Fungilab S.A., Barcelona, España) utilizando un spin R2 a $60 \mathrm{rpm}$ a $20^{\circ} \mathrm{C}$ y se expresó como $\mathrm{mPa}$.s, 
la densidad se midió con un picnómetro a $20^{\circ} \mathrm{C}$ y la unidad de medida fue $\mathrm{g} / \mathrm{mL}$, los sólidos solubles totales (SST) se midieron en ${ }^{\circ}$ Brix con un refractómetro digital (Atago Company Ltd, Tokio, Japón). La acidez total se midió por titulación con $\mathrm{NaOH}$ 0,1 N y se expresó como g de ácido láctico/100mL. Además, se realizó un análisis químico proximal de la bebida inmediatamente después de su obtención en el Laboratorio de Bromatología de la Universidad de Nariño.

Determinación de ácido ascórbico (AA). Se realizó utilizando una adaptación del método de Caro et al. (2004) y Nour et al. (2010). Muestras de bebida y ácido fosfórico se agitaron por $30 \mathrm{~min}$ a $4^{\circ} \mathrm{C}$, se centrifugaron a $6000 \mathrm{rpm}$, el sobrenadante se filtró en discos GHP (Acrodisc, Pall) de $13 \mathrm{~mm}$ $\mathrm{x} 0,45 \mu \mathrm{m}$ y finalmente, el ácido ascórbico fue determinado utilizando un cromatógrafo líquido de alta resolución (Waters $®$, Millford, MA, USA) equipado con una columna C18 (2) Luna Phenomenex (Waters®) de $250 \mathrm{~mm}$ x 4,6mm. Se empleó como fase móvil buffer fosfato de potasio monobásico a $25 \mathrm{mM}$, con un $\mathrm{pH}$ de 2,5 y un flujo de $0,7 \mathrm{~mL} / \mathrm{min}$. El eluido fue detectado utilizando un detector PDA (Waters®) 2998 a 210 y 254nm. La identificación de ácido ascórbico se realizó mediante comparación con el tiempo de retención de una solución patrón de ácido ascórbico analizada bajo las mismas condiciones. Para la cuantificación se emplearon relaciones de área cromatográfica con la solución patrón de ácido ascórbico. Los resultados fueron expresados como mg AA/100mL.

Determinación de fenoles totales. La determinación de fenoles totales en la bebida se realizó utilizando el método de Folin-Ciocalteu (Piljac-Žegarac et al., 2009). Una alícuota de $0,5 \mathrm{ml}$ de la bebida se mezcló con $0,5 \mathrm{ml}$ de reactivo de Folin-Ciocalteu y $10 \mathrm{ml}$ de una solución saturada de $\mathrm{Na}_{2} \mathrm{CO}_{3}$. Las muestras se mantuvieron a temperatura ambiente durante $1 \mathrm{~h}$. Posteriormente, se midió la absorbancia a $765 \mathrm{~nm}$ utilizando un espectrofotómetro (Thermo scientific, Madison, Wisconsin, USA). Las concentraciones se determinaron comparando la absorbancia de las muestras con una curva de calibración construida con 0, 50, 100, 150, 250 y 500mg de ácido gálico/L. Los resultados se expresaron como mg equivalentes de ácido gálico (GAE)/100mL.

Determinación de carotenoides. Para la determinación de carotenoides en las muestras de bebida se adaptó el método de Siong y Chin (1992). Se realizó extracción líquido-líquido, utilizando como solvente una mezcla de etanol-hexano. Posteriormente, se adicionó una solución saturada de $\mathrm{NaCl}$ al $10 \%$ y agua tipo I. El extracto orgánico se concentró con nitrógeno a sequedad y se adicionaron $2 \mathrm{~mL}$ de metanol grado HPLC. Las muestras se conservaron bajo oscuridad a $-18^{\circ} \mathrm{C}$ hasta el análisis. Previamente a la inyección, las muestras se diluyeron en una solución de acetonitrilo-metanolacetato de etilo. La identificación y cuantificación de carotenoides se realizó a través de cromatografía líquida de alta resolución empleando un detector PDA (Waters®) 2998 a 450nm y una columna Spherisob 0DS- C18 (Waters ${ }^{\circledR}$ ) de $5 \mu \mathrm{m}, 100 \mathrm{~mm}$ $\mathrm{x} 4,6 \mathrm{~mm}$. La fase móvil fue metanol con un flujo de $0,7 \mathrm{~mL} / \mathrm{min}$. La identificación de $\beta$-caroteno se realizó mediante comparación con el tiempo de retención de una solución patrón de éste compuesto analizada bajo las mismas condiciones. Para la cuantificación se emplearon relaciones de área cromatográfica con la solución patrón de $\beta$-caroteno. Los resultados se expresaron como $\mu \mathrm{g}$ $\beta$-caroteno $/ 100 \mathrm{~mL}$.

Capacidadantioxidante. Laactividad antirradical de la bebida se determinó mediante el ensayo de 2,2 difenil-1-picrilhidracil (DPPH) reportado por Morales de la Peña et al. (2010). Alícuotas de $0,1 \mathrm{~mL}$ de la bebida obtenida se mezclaron con $3,9 \mathrm{~mL}$ DPPH en metanol $(0,025 \mathrm{~g} / \mathrm{L})$ y $0,090 \mathrm{~mL}$ de agua destilada. El homogeneizado se agitó vigorosamente y se mantuvo en la oscuridad durante $30 \mathrm{~min}$. La absorción de las muestras se midió utilizando un espectrofotómetro (Thermo scientific, Madison, Wisconsin, USA) a $515 \mathrm{~nm}$ frente a un blanco de metanol sin DPPH. Los resultados se expresaron como trolox mmol/L. 
Diseño experimental y análisis estadístico. Los resultados de la determinación de las propiedades fisicoquímicas, el contenido de ácido ascórbido, fenoles totales, carotenides y la capacidad antioxidante de la bebida mixta de leche y tomate de árbol, se presentan como la media \pm desviación estándar de al menos tres determinaciones para cada evaluación. El análisis de resultados se desarrolló utilizando el software Statgraphics Centurion XV.II . Se empleó el diseño experimental Completamente al Azar y se aplicó el Análisis de Varianza (ANDEVA) seguido de la prueba de Rangos Múltiples (LSD de Fisher) a los resultados obtenidos para verificar si hubo diferencias significativas $(\mathrm{P}<0,05)$ en la concentración de compuestos bioactivos en la bebida mixta durante el tiempo de almacenamiento.

\section{RESULTADOS Y DISCUSIÓN}

Propiedades fisicoquímicas de la bebida. De acuerdo con los resultados de la Tabla 1, que pre- senta el análisis fisicoquímico de la bebida, tomando como referencia la NTC 512-2 (ICONTEC, 2007) de rotulado nutricional de alimentos envasados, se establece que una porción de $200 \mathrm{~mL}$ de bebida puede ser considerada como alta en ácido ascórbico debido a que dicho volumen de consumo habitual de referencia, aporta el $45,4 \%$ de esta vitamina a la dieta y es buena fuente de fibra, ya que contiene más del $10 \%$ del valor de referencia declarado en la norma técnica.

Los valores de SST, pH y acidez de la bebida cumplen con los exigidos por la NTC 5468 (ICONTEC, 2012) para zumos (jugos), néctares, purés (pulpas) y concentrados de frutas y la Resolución 7992 de 1991 del Ministerio de Salud de Colombia para néctares de fruta pasteurizados. Además, los SST, la densidad y la viscosidad se encuentran dentro de los rangos reportados en las investigaciones de Salvia et al. (2011) y Zulueta et al. (2007).

Tabla 1. Análisis fisicoquímico de la bebida de leche y tomate de árbol.

\begin{tabular}{lrc}
\hline \multicolumn{1}{c}{ Parámetro } & \multicolumn{1}{c}{ Valor } & $\begin{array}{c}\text { Contribución a la dieta según } \\
\text { el valor de referencia diario } \\
\text { de la NTC 512-2 (\%) }\end{array}$ \\
\hline Sólidos solubles totales a $20^{\circ} \mathrm{C}$ (-Brix) & $12,00 \pm 0,0$ & - \\
$\mathrm{pH}$ a $10,2^{\circ} \mathrm{C}$ & $4,19 \pm 0,02$ & - \\
Acidez (g ácido láctico/100 g) & $0,65 \pm 0,094$ & - \\
Densidad a $20^{\circ} \mathrm{C}(\mathrm{g} / \mathrm{mL})$ & $1,05 \pm 0,005$ & - \\
Viscosidad a $20^{\circ} \mathrm{C}(\mathrm{mPa} . \mathrm{s})$ & $39,92 \pm 0,73$ & - \\
Humedad $(\mathrm{g} / 100 \mathrm{~g})$ & $85,10 \pm 0,00$ & - \\
Cenizas $(\mathrm{g} / 100 \mathrm{~g})$ & $0,31 \pm 0,13$ & 7,6 \\
Proteína $(\mathrm{g} / 100 \mathrm{~g})$ & $1,80 \pm 0,01$ & 6,5 \\
Carbohidratos totales $(\mathrm{g} / 100 \mathrm{~g})$ & $9,30 \pm 0,54$ & 10,9 \\
Calcio $(\mathrm{mg} / 100 \mathrm{~g})$ & $52,13 \pm 0,80$ & 10,3 \\
Fósforo $(\mathrm{mg} / 100 \mathrm{~g})$ & $49,07 \pm 1,82$ & 9,0 \\
Potasio $(\mathrm{mg} / 100 \mathrm{~g})$ & $151,33 \pm 12,50$ & 0,82 \\
Hierro $(\mathrm{mg} / 100 \mathrm{~g})$ & $0,07 \pm 0,02$ & 45,4 \\
Ácido Ascórbico $(\mathrm{mg} / 100 \mathrm{~mL})$ & $12,97 \pm 0,603$ & 10,3 \\
Fibra cruda $(\mathrm{g} / 100 \mathrm{~g})$ & $1,22 \pm 0,12$ & - \\
$\beta$-caroteno $(\mu \mathrm{g} / 100 \mathrm{~mL})$ & $243,00 \pm 5,66$ & - \\
\hline
\end{tabular}


La Figura $1 \mathrm{~A}$ muestra que durante todo el almacenamiento, el pH y los sólidos solubles de la bebida se mantuvieron constantes. Además, se presentó un aumento significativo $(\mathrm{P}<0,05)$ de la acidez en

A

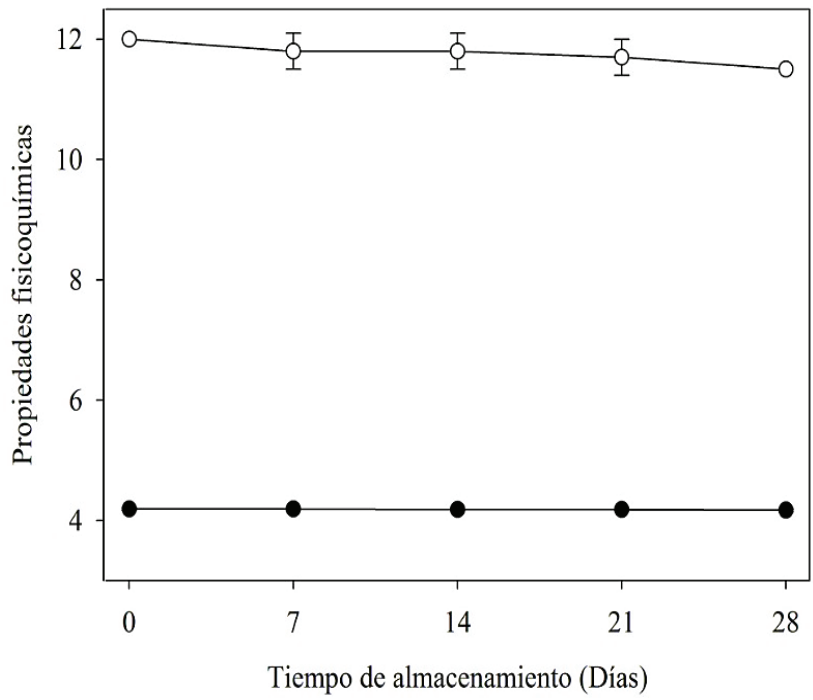

la bebida durante la primera semana de almacenamiento y después, este parámetro se mantuvo constante hasta el final del periodo evaluado (Figura 1B).

B

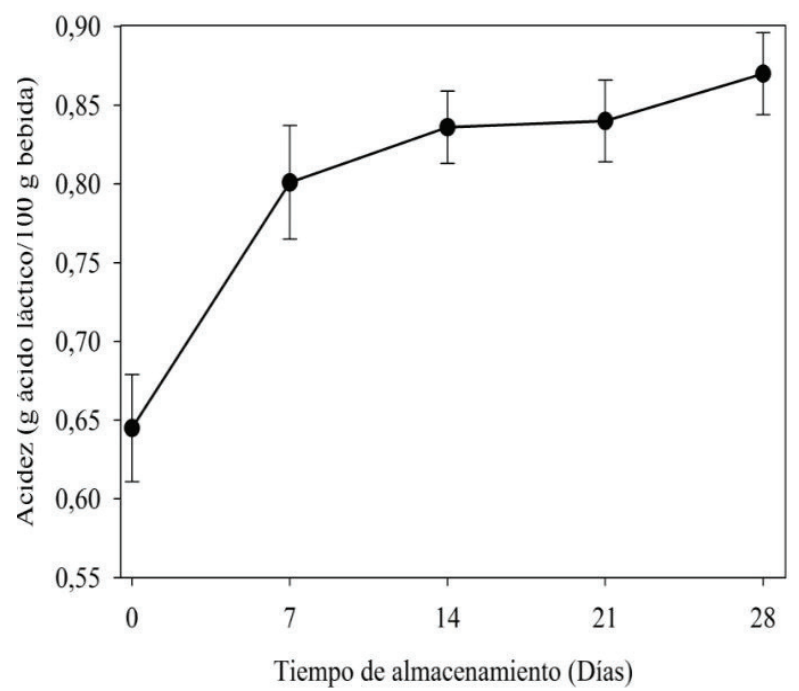

Figura 1. Comportamiento de las propiedades fisicoquímicas de la bebida mixta de leche y tomate de árbol durante el almacenamiento a $4^{\circ} \mathrm{C}:$ A) pH (•) y SST (०); B) acidez.

Similares resultados fueron reportados por Salvia et al. (2011), Morales de la Peña et al. (2010) y Vervoort et al. (2011), encontrando que los sólidos solubles totales y el pH se mantuvieron prácticamente constantes a lo largo del tiempo de almacenamiento a $4^{\circ} \mathrm{C}$ en bebidas de leche con jugos de frutas (naranja, kiwi, mango y piña) y en jugo de naranja.

\section{Análisis de la concentración de ácido ascórbi-} co. La concentración inicial de ácido ascórbico en la bebida mixta fue de $19,433 \pm 0,404 \mathrm{mg} / 100 \mathrm{~mL}$. Después del tratamiento térmico, la retención de

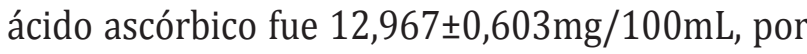
lo tanto, disminuyó en un $67 \%$. El contenido de este compuesto bioactivo en la bebida después de la pasteurización está dentro del rango de concentración (9,32 a 53,9mg/100mL) reportado por $\mathrm{Zu}$ lueta et al. (2007) en bebidas comerciales a base de leche y zumos de frutas. Sin embargo, como se muestra en la Figura 2, a partir de la primera semana disminuyó significativamente $(\mathrm{P}<0,05)$ y la concentración se ubicó por debajo del valor mínimo reportado en dicho estudio.

La retención de vitamina $\mathrm{C}$ se ha utilizado como un indicador de la calidad de los zumos de frutas y también como un marcador de su vida útil (Klimczak et al., 2007). De acuerdo con algunos autores, los zumos de fruta llegan al final de su vida útil en el punto en que la concentración de vitamina C llega al 50\% del contenido inicial (Shaw, 1992). La bebida de leche con tomate de árbol alcanzó este nivel a los siete días de almacenamiento. Este tiempo se encuentra por debajo de los tiempos reportados en otros estudios, debido a las diferencias en las condiciones (equipos) y variables (tiempo y temperatura) en las que se desarrolló el tratamiento de conservación. 


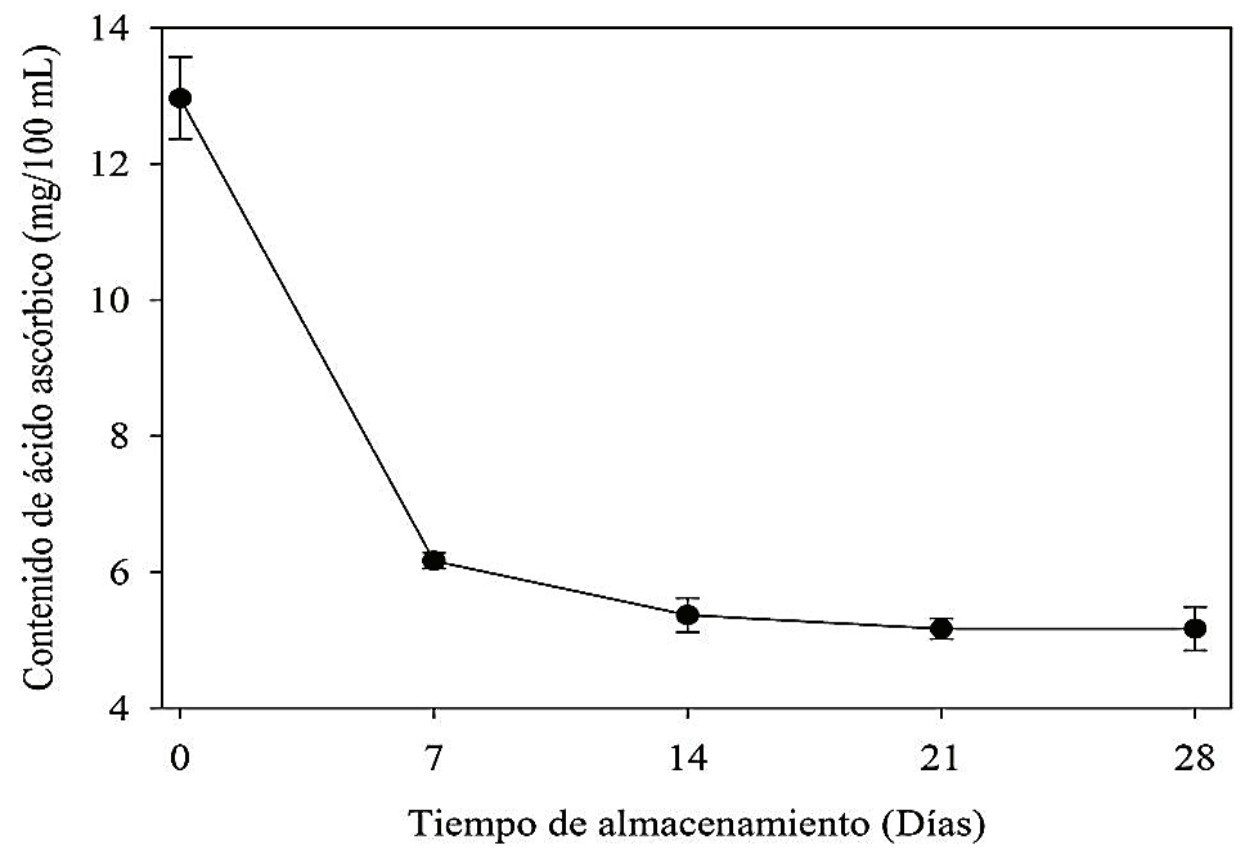

Figura 2. Variación del contenido de ácido ascórbico de la bebida mixta de leche y tomate de árbol durante el almacenamiento a $4^{\circ} \mathrm{C}$.

En la investigación de Morales de la Peña et al. (2010) se encontró que la concentración de vitamina $\mathrm{C}$ se redujo alrededor del $50 \%$ después de 17 días de almacenamiento a $4^{\circ} \mathrm{C}$. Torregrosa et al. (2006) señalan que en los jugos de naranja y zanahoria pasteurizados fue de 11 y 5 días, respectivamente y el trabajo de Yeom et al. (2000) en jugo de naranja pasteurizado, reporta una vida útil de 31 días.

La tendencia de disminución significativa $(\mathrm{p}<0,05)$ del contenido de ácido ascórbico durante la primera semana de almacenamiento refrigerado se observó en estudios realizados con bebidas de lechey jugos de fruta, por Morales de la Peña et al. (2010) y Zulueta et al. (2010). Piljac-Žegarac et al. (2009) señalan que el contenido de vitamina $C$ en zumo de arándano se redujo a cero después de 7 días de almacenamiento, y se redujo a $50 \%$ del valor inicial dentro de las primeras $74 \mathrm{~h}$. En otros estudios (Laorko et al., 2013; Choi et al., 2002), se reseña que el contenido de vitamina $C$ en jugo de piña $y$ naranja disminuyó considerablemente $(p<0,05)$ durante el primer mes del almacenamiento refrigerado y se encontró que más del 50\% del ácido ascórbico se perdió durante las tres primeras semanas y se degradó completamente después de cinco semanas de almacenamiento.

La disminución del ácido ascórbico se podría atribuir a su termolabilidad, siendo susceptible a la oxidación química y enzimática, y su velocidad de deterioro depende del método de procesamiento empleado y las condiciones de almacenamiento. Los factores que determinan la estabilidad de este compuesto bioactivo en una matriz alimenticia son la temperatura, tiempo, disponibilidad de oxígeno, pH, luz y la presencia de otros agentes antioxidantes y reductores (Zerdin et al., 2003; Rawson et al., 2011; Rodríguez et al., 2015).

\section{Análisis de la concentración de fenoles tota-} les. Tal como lo muestra la Figura 3, el contenido de fenoles totales en la bebida presentó una disminución significativa $(\mathrm{p}<0,05)$ durante el almacenamiento. 


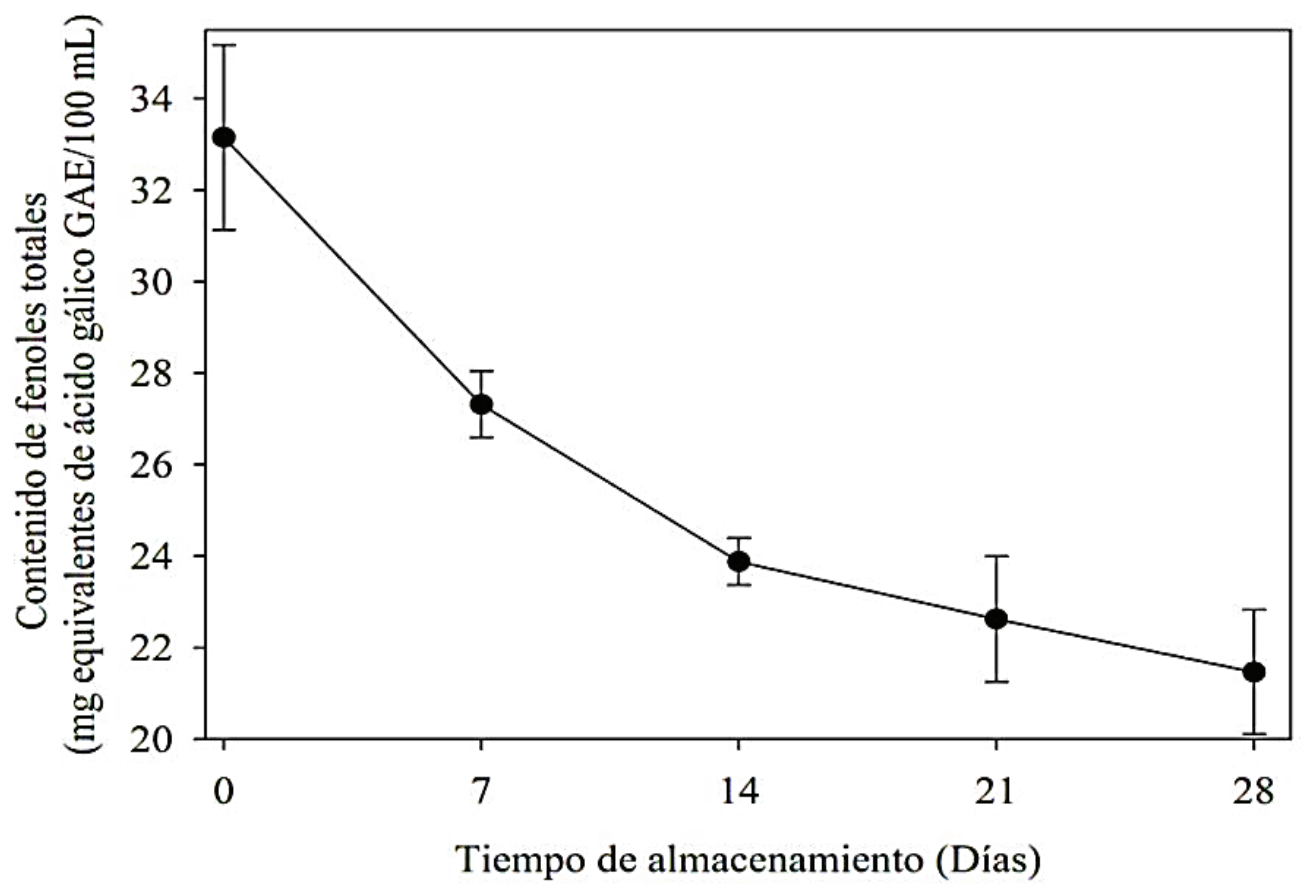

Figura 3. Variación del contenido de fenoles totales de la bebida mixta de leche y tomate de árbol durante el almacenamiento a $4^{\circ} \mathrm{C}$.

El contenido máximo de fenoles totales obtenido en esta investigación, se ubicó dentro del rango reportado en el estudio de Zulueta et al. (2007). A partir de la segunda semana de almacenamiento, se presentó una disminución de la cantidad de estos compuestos por debajo del valor mínimo observado en dicho estudio. Similares resultados fueron registrados por Morales de la Peña et al. (2010).

Las diferencias en el contenido fenólico total entre la bebida de leche y tomate de árbol y las bebidas estudiadas por Zulueta et al. (2007), son atribuidas principalmente al tipo de fruta, el grado de maduración, las condiciones ambientales de cultivo y cosecha de las frutas empleadas para su elaboración, así como a los procesos tecnológicos utilizados para su obtención, al tiempo y condiciones de almacenamiento (Morales de la Peña et al., 2010; Klimczak et al., 2007).

Se presume que los tratamientos térmicos son por lo general, la causa principal de la disminución de los antioxidantes naturales (Morales de la Peña et al., 2010). Las altas temperaturas aplicadas durante el proceso podrían haber afectado los compuestos fenólicos susceptibles en la bebida haciéndolos fácilmente degradables en el tiempo. Adicionalmente, Cao et al. (2012), Chandrasekara y Shahidi (2012) señalan que la disminución de fenoles totales se debe a la degradación por oxidación y su polimerización con proteínas.

Los antioxidantes fenólicos que se encuentran en el tomate de árbol, son principalmente antocianinas tales como: delfinidinas, cianidina y pelargonidina (Osorio et al., 2012).

Análisis de la concentración de carotenoides. Como se observa en la Figura 4, el contenido de $\beta$-caroteno disminuyó con el tiempo de almacenamiento. La concentración inicial de carotenoides en la bebida mixta fue $278,00 \pm 7,55 \mu \mathrm{g}$ de $\beta$-caroteno $/ 100 \mathrm{~mL}$. Después de aplicar el tratamiento térmico, éste decreció significativamente $(\mathrm{p}<0,05)$. 


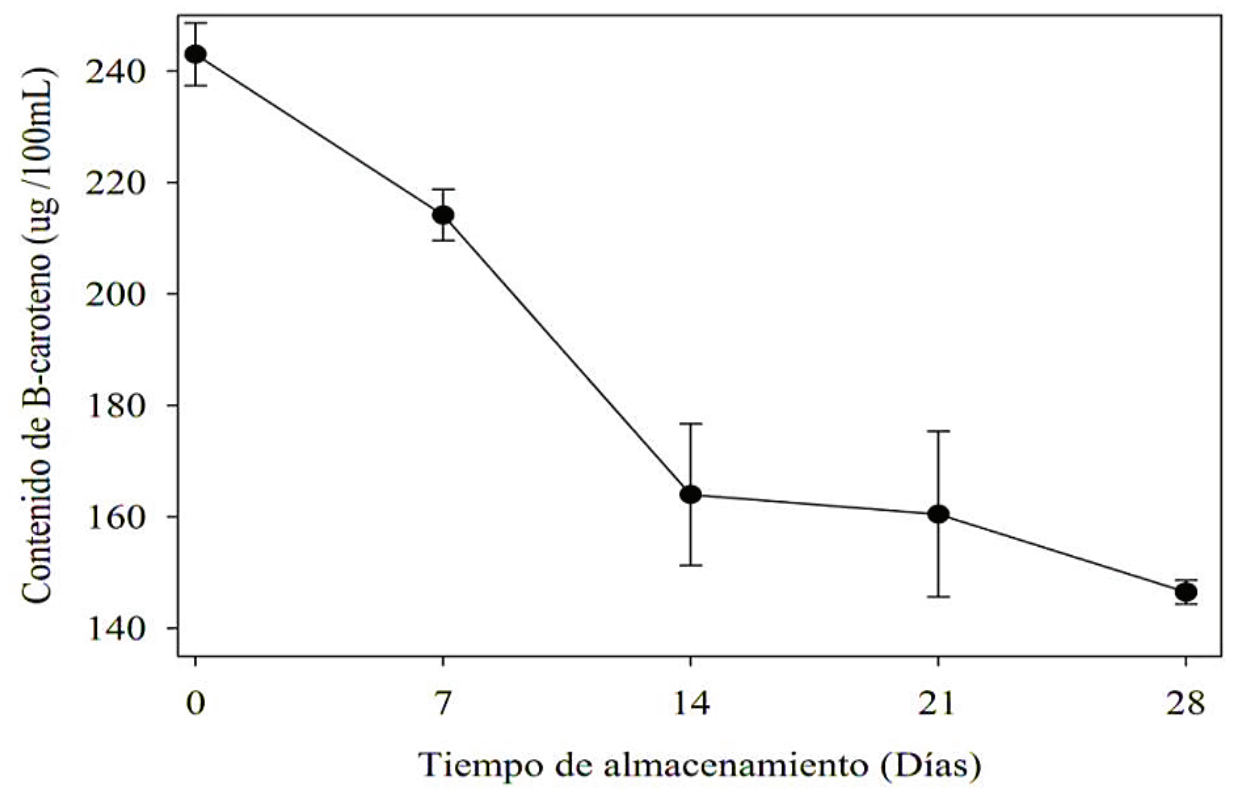

Figura 4. Variación del contenido de $\beta$-caroteno en la bebida mixta de leche y tomate de árbol durante el almacenamiento a $4^{\circ} \mathrm{C}$.

El contenido de $\beta$-caroteno obtenido hasta la segunda semana de almacenamiento $(164 \mu \mathrm{g}$ de $\beta$-caroteno $/ 100 \mathrm{~mL}$ ) fue superior al rango reportado en el estudio de Zulueta et al. (2007). Similares resultados a los obtenidos en esta investigación fueron encontrados por Morales de la Peña et al. (2010), observando que el contenido total de carotenoides de bebidas de leche de soja y jugos de frutas tendió a disminuir a medida que se incrementó el tiempo de almacenamiento. Hsu et al. (2008) observaron que durante los primeros 14 días de almacenamiento a $4^{\circ} \mathrm{C}$, los carotenoides totales y el licopeno de zumo de tomate procesado térmicamente se degradó rápidamente en 16 y 12\% en comparación con el control, y no hubo una pérdida significativa durante el almacenamiento entre los días 14 a 28.

Cortés et al. (2006) y Odriozola et al. (2009), encontraron que la concentración total de carotenoides en zumos de tomate y naranja tratados con pulsos eléctricos y térmicamente, disminuyó durante el tiempo de almacenamiento refrigerado, independientemente del tratamiento de conservación aplicado. Rattanathanalerk et al. (2009), Zepka y
Mercadante (2009) y Aramwit et al. (2010), investigaron el efecto de la pasteurización sobre los zumos de mora, piña y manzana, concluyendo que este tratamiento conduce a una disminución en los niveles de carotenoides.

Se presume que la labilidad térmica de éstos compuestos bioactivos parece estar influenciada por las condiciones experimentales y la naturaleza de la matriz del alimento. La degradación de los carotenoides sucede por reacciones de oxidación e isomerización y se atribuye principalmente a la intensidad del tratamiento térmico, la estructura química de las moléculas y la exposición del alimento al oxígeno (Mertz et al., 2010; Rawson et al., 2011).

En las muestras de bebida de leche y tomate deárbol a lo largo del tiempo de almacenamiento, se identificaron otros carotenos como $\beta$-criptoxantina, 9-cis- $\beta$-caroteno, 13 -cis- $\beta$-caroteno y licopeno. Según la cantidad relativa de cada uno de éstos, se establece que el $\beta$-Caroteno se encuentra en mayor proporción en comparación a los demás compuestos identificados, seguido por $\beta$-criptoxantina, 
13-cis- $\beta$-caroteno y 9 -cis- $\beta$-caroteno. Similares resultados se han reportado en las investigaciones de Mertz et al. (2010) y Rodríguez et al. (1983), obteniendo que los principales carotenoides del tomate de árbol son ésteres de trans- $\beta$-criptoxantina y trans- $\beta$-caroteno. Así mismo $\beta$-criptoxantina y $\beta$-caroteno son los compuestos de este tipo más importantes en la cáscara y la pulpa de tomate de árbol.

\section{Potencial antioxidante de la bebida de leche} y tomate de árbol. La capacidad antioxidante de la bebida estuvo en un rango entre $2,658 \pm 0,048$ y 3,450 $\pm 0,142$ Trolox $\mathrm{mmol} / \mathrm{L}$ durante el almacenamiento refrigerado. Estos valores se ubican dentro del rango (0,61 a 3,60 Trolox $\mathrm{mmol} / \mathrm{L}$ ) establecido por Zulueta et al. (2007).

Como se presenta en la Figura 5, la capacidad antioxidante total de la bebida mixta de leche y tomate de árbol durante el almacenamiento a $4^{\circ} \mathrm{C}$ disminuyó gradualmente y de forma significativa $(\mathrm{p}<0,05)$ a medida que se incrementó el tiempo de almacenamiento y al cabo de un mes, la pérdida de la capacidad antioxidante fue del $30 \%$.
Resultados semejantes reportaron Morales de la Peña et al. (2010), quienes encontraron que fue significativamente menor en las bebidas tratadas térmicamente. Piljac-Žegarac et al. (2009) estudiaron la reducción de la capacidad antioxidante del jugo de arándano encontrando que al final del período de almacenamiento (56 días) varió de $69 \%$ (sin procesar) a $52 \%$ (tratado con pulsos eléctricos) y $79 \%$ (procesado con altas presiones). Mientras que en jugos sin tratamiento previo se observó una disminución significativa de su capacidad antioxidante que varió de 15\% (en jugo de arándano) a 62\% (en jugo de cereza).

Zulueta et al. (2007) establecen que el porcentaje de jugo empleado en cada bebida analizada no influye significativamente en la capacidad antioxidante. En consecuencia, las diferencias entre las variables se deben a la composición del jugo (tipos de fruta) y las condiciones de almacenamiento y procesamiento, estableciendo que los tratamientos térmicos enérgicos son la principal causa de la reducción de los antioxidantes naturales.

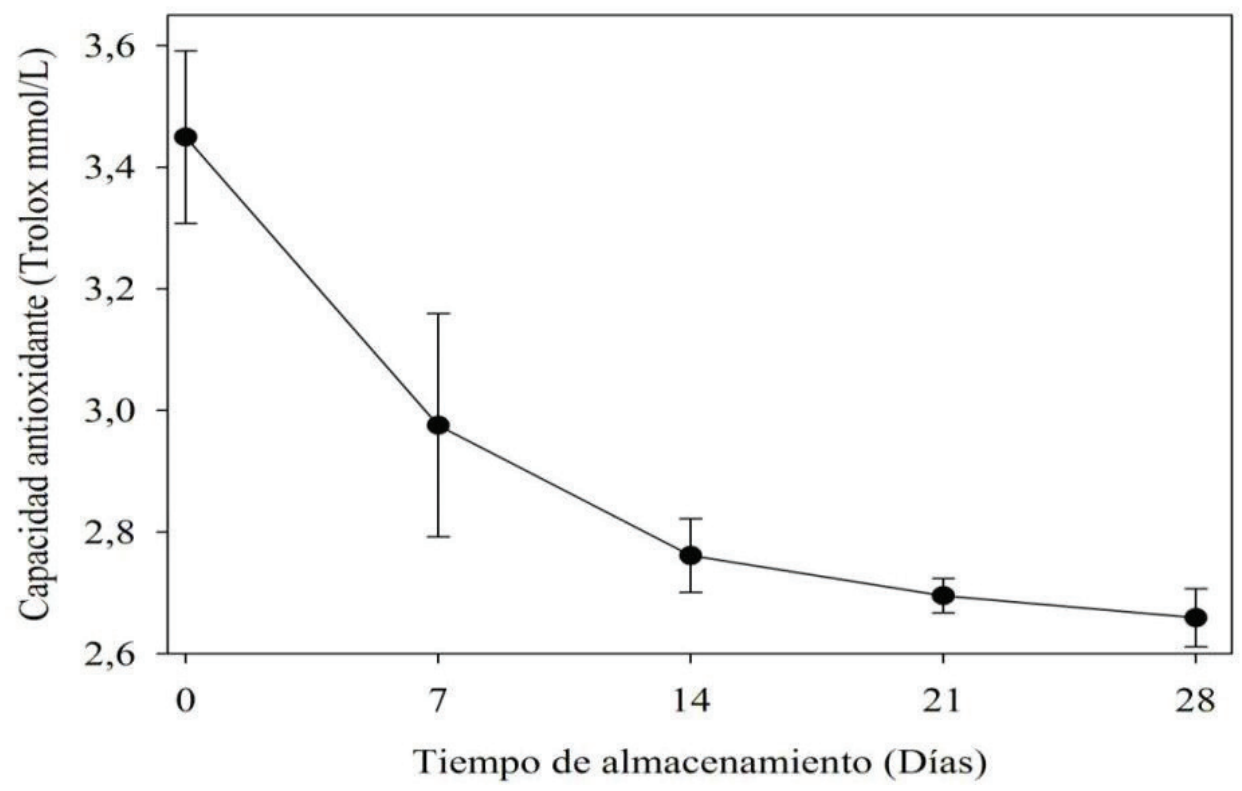

Figura 5. Variación de la capacidad antioxidante total de la bebida mixta de leche y tomate de árbol durante el tiempo de almacenamiento a $4^{\circ} \mathrm{C}$. 
Sin embargo, aunque las temperaturas de pasteurización más bajas reducen el abuso térmico del producto, obteniendo una mayor retención de componentes de color, sabor y una disminución en la producción de compuestos indeseables, éstas no inactivan lo suficiente a las enzimas de la matriz del alimento y permiten la generación de alteraciones en las propiedades fisicoquímicas y organolépticas del producto (Hsu et al., 2008). Por lo tanto, aunque se produzca pérdida de compuestos bioactivos y disminución de la calidad sensorial, los alimentos líquidos se han conservado tradicionalmente empleando tratamientos térmicos (Rodríguez et al., 2015).

En general, la capacidad antioxidante de los zumos de frutas se relaciona con la composición y la concentración de compuestos bioactivos tales como vitaminas, fenoles, carotenoides o flavonoides (Sánchez et al., 2006). Takeoka et al. (2001) reportan que la vitamina $\mathrm{C}$ y los fenoles son los principales componentes antioxidantes en zumos de frutas. Es así como en la bebida mixta compuesta por leche UHT descremada y tomate de árbol, la capacidad antioxidante se relacionó con el contenido de fenoles totales, ácido ascórbico y carotenoides. La disminución de estos compuestos generó un consecuente decrecimiento de la capacidad antioxidante. En la investigación de Morales de la Peña et al. (2010) se observó que los cambios en la capacidad antioxidante durante el tiempo, están asociados principalmente a las variaciones de contenido de vitamina $\mathrm{C}$.

Se reporta que la vitamina $\mathrm{C}$ es el principal compuesto bioactivo responsable de la capacidad antioxidante en bebidas de zumos de frutas con leche (Zulueta et al., 2007), encontrando que ésta contribuye entre 65 y $100 \%$ del potencial antioxidante de bebidas derivadas de cítricos, y los compuestos fenólicos parecen ser de los principales aportantes del potencial antioxidante de los zumos no cítricos; mientras que la contribución de los carotenoides es posiblemente insignificante (Gardner et al., 2000).
Desde el punto de vista nutricional y funcional, en el estudio de bebidas basadas en la mezcla de leche y jugos de frutas, es importante conocer la fracción bioaccesible de los compuestos bioactivos analizados como la vitamina $\mathrm{C}$, fenoles y carotenoides y determinar la influencia de la matriz del alimento en su bioaccesibilidad. Esta información aún es limitada y la capacidad antioxidante in vivo requiere mayor investigación (Rodríguez et al., 2014; 2015; Carranco et al., 2011).

\section{CONCLUSIONES}

La bebida mixta de leche y tomate de árbol presentó compuestos bioactivos que podrían otorgarle características funcionales. Durante la primera semana de almacenamiento a $4^{\circ} \mathrm{C}$ se encontraron los máximos niveles de ácido ascórbico, $\beta$-caroteno, fenoles totales y fibra. Además, la capacidad antioxidante de la bebida disminuyó significativamente junto con el contenido de fenoles totales, ácido ascórbico y carotenoides en el tiempo evaluado. El pH y los SST se mantuvieron constantes, mientras que la acidez aumentó significativamente durante la primera semana de almacenamiento. Considerar un tratamiento de conservación diferente al térmico, constituye una alternativa para garantizar una menor pérdida de compuestos bioactivos y favorecer el flavour de este tipo de productos.

\section{AGRADECIMIENTOS}

Los autores expresan sus sinceros agradecimientos a la Vicerrectoría de Investigaciones, Postgrados y Relaciones Internacionales y al personal del Laboratorio de Cromatografía de la Universidad de Nariño por hacer posible el desarrollo de este trabajo.

\section{REFERENCIAS BIBLIOGRÁFICAS}

ANDRÉS, V.; VILLANUEVA, M.; MATEOS, I.; TENORIO, M. 2014. Colour, bioactive compounds and antioxidant capacity of mixed beverages based on fruit juices with milk or soya. Journal of Food and Nutrition Research. 53(1):71 - 80 . 
ARAMWIT, P.; BANG, N.; SRICHANA, T. 2010. The properties and stability of anthocyanins in mulberry fruits. Food Research International. 43:1093 - 1097. doi:10.1016/j.foodres.2010.01.022.

BEECHER, G. 1998. Nutrient content of tomatoes and tomato products. Proceedings of the Society for Experimental Biology and Medicine. 218:98 - 100. doi:10.3181/00379727-218-44282a.

CAO, X.; BI, X.; HUANG, W.; WU, J.; HU, X.; LIAO, X. 2012. Changes of quality of high hydrostatic pressure processed cloudy and clear strawberry juices during storage. Innovative Food Science and Emerging Technologies. 16:181 - 190. doi:10.1016/j.ifset.2012.05.008.

CARO, A.; PIGA, A.; VACCA, V. 2004. Changes of flavonoids, vitamin $C$ and antioxidant capacity in minimally processed citrus segments and juices during storage. Food Chemistry. 84:99 - 105. doi:10.1016/S03088146(03)00180-8.

CARRANCO, M.E.; CALVO-CARRILLO, M.; PÉREZ-GIL, F. 2011. Carotenoides y su función antioxidante: Revisión. Archivos Latinoamericanos de Nutrición. 61:233 - 241.

CASTAÑEDA, C.; RAMOS, LL.; IBÁÑEZ, V. 2008. Evaluación de la capacidad antioxidante de siete plantas medicinales peruanas. Revista Horizonte Médico. 8:56 - 72.

CERVATO, G.; CAZZOLA, R.; CESTARO, B. 1999. Studies on the antioxidant activity of milk caseins. International Journal of Food Science and Nutrition. 50:291 - 296.

CORTÉS, C.; TORREGROSA, F.; ESTEVE, M.; FRÍGOLA A. 2006. Carotenoid profile modification during refrigerated storage in untreated and pasteurized orange juice and orange juice treated with high intensity pulsed electric fields. Journal of Agricultural and Food Chemistry. 54:6247 - 6254. doi: 10.1021/jf060995q.

CHANDRASEKARA, A.; SHAHIDI, F. 2012. Bioaccessibility and antioxidant potential of millet grain phenolics as affected by simulated in vitro digestion and microbial fermentation. Journal of Functional Foods. 4(1):226 - 237.

CHOI, M.; KIM, G.; LEE, H. 2002. Effect of ascorbic acid retention on juice color and pigment stability in blood orange (Citrus sinensis) juice during refrigerated sto- rage. Food Research International. 35:753 - 759. doi: 10.1016/S0963-9969(02)00071-6.

GARDNER, P.; WHITE, T.; MCPHAIL, D.; DUTHIE, G. 2000. The relative contributions of vitamin C, carotenoids and phenolics to the antioxidant potential of fruit juices. Food Chemistry. 68:471 - 474. doi: S03088146(99)00225-3.

HE, Z.; YUAN, B.; ZENG, M.; TAO, G.; CHEN, J. 2015. Effect of simulated processing on the antioxidant capacity and in vitro protein digestion of fruit juice-milk beverage model systems. Food Chem. 175:457 - 464. doi:10.1016/j.foodchem.2014.12.007.

HSU, K.; TAN, F.; CHI, H. 2008. Evaluation of microbial inactivation and physicochemical properties of pressurized tomato juice during refrigerated storage. Food Science and Technology. 41:367 - 375. doi:10.1016/j. lwt.2007.03.030.

ICONTEC-INSTITUTO COLOMBIANO DE NORMAS TÉCNICAS Y CERTIFICACIÓN. 2007. Rotulado o etiquetado. Parte 2: rotulado Nutricional de alimentos envasados. NTC 512-2. ICONTEC, Bogotá. 36 p.

ICONTEC-INSTITUTO COLOMBIANO DE NORMAS TÉCNICAS Y CERTIFICACIÓN. 2012. Jugo (zumo), pulpa, néctar de frutas y sus concentrados. NTC 5468. ICONTEC. Bogotá. 11 p.

KLIMCZAK, I.; MALECKA, M.; SZLACHTA, M.; GLISZCZYŃSKA, A. 2007. Effect of storage on the content of polyphenols, vitamin $C$ and the antioxidant activity of orange juices. Journal of Food Composition and Analysis. 20:313 - 322. doi:10.1016/j.jfca.2006.02.012.

LAORKO, A.; TONGCHITPAKDEE, S.; YOURAVONG, W. 2013. Storage quality of pineapple juice non-thermally pasteurized and clarified by microfiltration. Journal of Food Engineering. 116:554 - 561. doi: 10.1016/j.jfoodeng.2012.12.033.

LINDMARK, H.; AKESSON, B. 2000. Antioxidative factors in milk. British Journal of Nutrition. 84:103 - 110. MERTZ, C.; BRAT, P.; CARIS-VEYRAT, C.; GUNATA,Z. 2010. Characterization and thermal lability of carotenoids and vitamin $\mathrm{C}$ of tamarillo fruit (Solanum betaceum Cav.). Food Chemistry. 119:653 - 659. doi:10.1016/j.foodchem.2009.07.009. 
MINISTERIO DE SALUD COLOMBIA. 1991. Resolución 7992 (21, julio, 1991). Por la cual se reglamenta parcialmente el Título V de la Ley 09 de 1979 en lo relacionado con la elaboración, conservación y comercialización de Jugos. Concentrados, Néctares, Pulpas, Pulpas Azucaradas y Refrescos de Frutas. En: http://www.confia.com. co/normatividad/Resolucion $\% 207992 \% 20 \mathrm{de} \% 20$ 1991\%20Jugos\%20concentrados\%20Nectares $\% 20$ pulpas\%20refrescos.pdf; consulta: febrero, 2016.

MORALES DE LA PEÑA, M.; SALVIA, L.; ROJAS, M.; MARTÍN, 0. 2010. Impact of high intensity pulsed electric field on antioxidant properties and quality parameters of a fruit juice-soymilk beverage in chilled storage. Food Science and Technology. 43:872 - 881. doi:10.1016/j. lwt.2010.01.015.

NOUR, V.; TRANDAFIR, I.; IONICA, M. 2010. HPLC Organic Acid Analysis in different citrus juices under reversed phase Conditions. Notulae Botanicae Horti Agrobotanici Cluj-Napoca. 38:44 - 48. doi: 10.15835/ nbha3814569.

ODRIOZOLA, I.; SOLIVA, R.; HERNÁNDEZ, T.; MARTÍN, 0. 2009. Carotenoid and phenolic profile of tomato juices processed by high intensity pulsed electric fields compared with conventional thermal treatments. Food Chemistry. 112:258 - 266. doi:10.1016/j.foodchem.2008.05.087.

OSORIO, C.; HURTADO, N.; DAWID, C.; HOFMANN, T.; HEREDIA, F; MORALES, A. 2012. Chemical characterisation of anthocyanins in tamarillo (Solanum betaceum Cav.) and Andes berry (Rubus glaucus Benth.) fruits. Food Chemistry. 132:1915 - 1921. doi:10.1016/j.foodchem.2011.12.026.

PILJAC-ŽEGARAC, J.; VALEK, L.; MARTINEZ, S.; BELŠČAK, A. 2009. Fluctuations in the phenolic content and antioxidant capacity of dark fruit juices in refrigerated storage. Food Chemistry. 113:394 - 400. doi:10.1016/j. foodchem.2008.07.048.

PSZCZOLA, D.E. 2005. Ingredients. Making fortification. Food Technology. 59:44 - 61.

RATTANATHANALERK, M.; CHIEWCHAN, N.; SRICHUMPOUNG, W. 2009. Effect of thermal Processing on the Quality Loss of Pineapple Juice. Journal of Food Engineering. 66:259 - 265. doi:10.1016/j.jfoodeng.2004.03.016.
RAWSON, A.; PATRAS, A.; TIWARI, B.; NOCI, F.; KOUTCHMA, T.; BRUNTON, N. 2011. Effect of thermal and non thermal processing technologies on the bioactive content of exotic fruits and their products: Review of recent advances. Food Research International. 44:1875 - 1887. doi:10.1016/j.foodres.2011.02.053.

RODRIGUEZ, D.; BOBBIO, P.; BOBBIO, F. 1983. Carotenoid composition and vitamin A value of the Brazilian fruit Cyphomandra betacea. Food Chemistry. 12:61 - 65.

RODRÍGUEZ, M.J.; ROJAS, M.A.; ELEZ, P.; MARTÍN, 0. 2014. In vitro bioaccessibility of health-related compounds as affected by the formulation of fruit juice- and milk-based beverages. Food Res. Int. 62:771 - 778. doi: dx.doi.org/10.1016/j.foodres.2014.04.037.

RODRÍGUEZ, M.J.; ANCOS, B.; SÁNCHEZ, C.; CANO, M.P.; ELEZ, P.; MARTÍN, 0. 2015. Impact of food matrix and processing on the in vitro bioaccessibility of vitamin C, phenolic compounds, and hydrophilic antioxidant activity from fruit juice-based beverages. J. Funct. Foods. 14:33 - 43. doi: dx.doi.org/10.1016/j.jff.2015.01.020.

SALVIA, L.; MORALES, M.; ROJAS, M.; MARTÍN, 0. 2011. Microbial and enzymatic stability of fruit juice-milk beverages treated by high intensity pulsed electric fields or heat during refrigerated storage. Food Control. 22:1639 - 1646. doi:10.1016/j.foodcont.2011.03.022.

SÁNCHEZ, C.; PLAZA, L.; DE ANCOS, B.; CANO, M. 2006. Impact of high-pressure and traditional thermal processing of tomato puree on carotenoids, vitamin $C$ and antioxidant activity. Journal of the Science of Food and Agriculture. 86:171 - 179. doi:10.1002/jsfa.2321.

SATUÉ, M.; FRANKEL, E.; RANGAVAIJHYALA, N.; GERMAN, J. 2000. Lactoferrin in infant formulas: effect on oxidation. Journal of Agricultural and Food Chemistry. 48:4984 - 4990. doi: 10.1021/jf0002490.

SHAW, P.E. 1992. Shelf-life and aging of citrus juices, juice drinks and related soft drinks, pp. 173 - 199. En: Redd, J. B., Shaw, P. E., Hendrix, C.M., Hendrix, D.L. Quality control manual for citrus processing plants. Agscience Press. Florida, Estados Unidos.

SIONG, T.; CHIN, L. 1992. Analysis of Carotenoids in Vegetables by HPLC. ASEAN Food Journal. 7:91 - 99. 
TAKEOKA, G.; DAO, L.; FLESSA, S.; GILLESPIE, D.; JEWELL, W.; HUEBNER, B. 2001. Processing effects on lycopene content and antioxidant activity of tomatoes. Journal of Agricultural and Food Chemistry. 49:3713 3717. doi: 10.1021/jf0102721.

TORREGROSA, F.; ESTEVE, M.; FRÍGOLA, A.; CORTÉS, C. 2006. Ascorbic acid stability during refrigerated storage of orange juice treated by high pulsed electric field and comparison with pasteurized juice. Journal of Food Engineering. 73:339 - 345. doi:10.1016/j.jfoodeng.2005.01.034.

VERVOORT, L.; VAN DER PLANCKEN, I.; GRAUWET, T.; TIMMERMANS, R.; MASTWIJK, H.; MATSER, A. 2011. Comparing equivalent thermal, high pressure and pulsed electric field processes for mild pasteurization of orange juice Part II: Impact on specific chemical and biochemical quality parameters. Innovative Food Science and Emerging Technologies. 12:466 - 477. doi:10.1016/j.ifset.2011.06.003.

YEOM, H.; STREAKER, C.; ZHANG, Q.; MIN, D. 2000. Effects of pulsed electric fields on the quality of orange juice and comparison with heat pasteurization. Journal of Agricultural and Food Chemistry. 48:4597 - 4605.

ZEPKA, L.; MERCADANTE, A. 2009. Degradation compounds of carotenoids formed during heating of a simulated cashew apple juice. Food Chemistry. 117:28 - 34. doi:10.1016/j.foodchem.2009.03.071.

ZERDIN, K.; ROONEY, M.; VERMUE, J. 2003. The vitamin $\mathrm{C}$ content of orange juice packed in an oxygen scavenger material. Food Chemistry. 82:387 - 395. doi:10.1016/ S0308-8146(02)00559-9.

ZULUETA, A.; ESTEVE, M.; FRASQUET, I.; FRÍGOLA, A. 2007. Vitamin C, vitamin A, phenolic compounds and total antioxidant capacity of new fruit juice and skim milk mixture beverages marketed in Spain. Food Chemistry. 103: 1365 - 1374. doi:10.1016/j.foodchem.2006.10.052.

ZULUETA, A.; ESTEVE, M.; FRÍGOLA, A. 2010. Ascorbic acid in orange juice-milk beverage treated by high intensity pulsed electric fields and its stability during storage. Innovative Food Science and Emerging Technologies. 11:84 - 90. doi:10.1016/j.ifset.2009.07.007. 